\title{
Prof. Dr. Nina Dethloff, Universität Bonn
}

\author{
Juristinnen machen Karriere - wir stellen sie vor
}

Das Interview führte djb-Präsidentin Ramona Pisal am 16. Oktober 2012.

Liebe Frau Professorin Dethloff, warum haben Sie sich nach dem Abitur für das Jurastudium entschieden?

Ich war schon immer sehr an Menschen und an Sprachen interessiert, aber mir war zunächst nicht klar, was ich damit später praktisch anfangen sollte. Ich habe mich dann für das Jurastudium entschieden, ohne recht zu wissen, was mich erwartete. Die ersten Semester waren mehr von Pflichterfüllung als von Freude am Stoff geprägt. Aber als ich einen Überblick gewonnen hatte, hat mich mein Fach begeistert. Leider ist der Zugang zum Jurastudium dadurch erschwert, dass die Schule keine Vorstellung davon vermittelt, was Jura bedeutet. Auch darum habe ich mich für Arbeitsgemeinschaften Jura in den Schulen engagiert und meine wissenschaftlichen Assistenten und Assistentinnen dazu ermutigt. Diese Initiative zeigt Erfolg, denn wir haben damit viele wirklich interessierte junge Menschen für das Jurastudium gewinnen können, die eine Vorstellung davon haben, was sie erwartet.

Nach den ersten Semestern in Ihrer Heimatstadt Hamburg hat es Sie bald nach Genf gezogen und später nach Freiburg. War der Studienortwechsel eine Bereicherung?

Absolut ja. Damals gab es noch nicht die Möglichkeit, schon während des Studiums mit Erasmus-Programmen oder Ähnlichem in die verschiedensten europäischen Länder zu gehen. Aber der DAAD bot Stipendien an, um ein Studienjahr in Genf oder Lausanne zu verbringen. Meine französischen Sprachkenntnisse waren schon gut, ich entschied mich für Genf und habe dort eine wunderbare Zeit erlebt, bin in das Studium richtiggehend eingetaucht und habe für mich entdeckt, was mich bis heute interessiert, fasziniert und meine beruflichen Entscheidungen maßgeblich geprägt hat: das Kennenlernen anderer Rechtsordnungen und die Rechtsvergleichung, wie und vor welchen politischen, sozialen und kulturellen Hintergründen für vergleichbare Probleme in den verschiedenen Ländern unterschiedliche Lösungen entwickelt worden sind. Und ich habe gemerkt, dass ich große Freude daran habe, den Dingen auf den Grund zu gehen, mehr zu erfahren, mich intensiv mit einem Gebiet zu beschäftigen.

Nach dem 1. Staatsexamen in Freiburg haben Sie Deutschland erst einmal den Rücken gekehrt. Damals war es noch nicht üblich, im englischsprachigen Ausland den LL.M. zu machen. Was hatte Sie nach Washington geführt?

Ich hatte damals als Fulbright-Stipendiatin verschiedene Optionen für ein USA-Studium. Für Washington und die Georgetown University habe ich mich entschieden, weil es ungemein spannend war, im Zentrum der Macht, einer Weltmacht, mitzuerleben, wie Recht gestaltet wird. Neben den Vorlesungen,
Nina Dethloff, geboren 1958 in Hamburg, studierte von 1976 bis 1982 Rechtswissenschaften in Hamburg, Genf und Freiburg. Die Erste Juristische Staatsprüfung bestand sie 1982. Im Anschluss, von 1982 bis 1997, arbeitete sie als Wissenschaftliche Mitarbeiterin am Institut für ausländisches und internationales Privatrecht an der Universität Freiburg. Von 1983 bis 1984 studierte sie an der Georgetown University in Washington, D.C./USA und schloss ihr Studium mit dem Master of Laws (LL.M.) ab. Von 1986 bis 1987 war sie als Beraterin bei der Federal Trade Commission, Washington, D.C. tätig. 1987 wurde sie sodann als Attorney at Law für den Staat New York zugelassen.

Den Juristischen Vorbereitungsdienst, den sie 1991 mit der Zweiten Juristischen Staatsprüfung beendete, absolvierte sie von 1988 bis 1991. Ebenfalls 1991 wurde sie durch die Rechtswissenschaftliche Fakultät der Universität Freiburg mit der Dissertation „Die einverständliche Scheidung“" (summa cum laude) promoviert.

2000 folgte ihre Habilitation an der Rechtswissenschaftlichen Fakultät der Universität Freiburg mit der Schrift „Die Europäisierung des Wettbewerbsrechts“. Sie erwarb damit die Lehrbefugnis für die Fächer Bürgerliches Recht, Internationales Privatrecht, Rechtsvergleichung und Europäisches Privatrecht. Seit dem Sommersemester 2001 ist sie Inhaberin des Lehrstuhls für Bürgerliches Recht, Internationales Privatrecht, Rechtsvergleichung und Europäisches Privatrecht und seit 2003 Direktorin des Instituts für Deutsches, Europäisches und Internationales Familienrecht der Universität Bonn.

Nina Dethloff hat seit 2001 an zahlreichen Anhörungen als Sachverständige vor dem Rechtsausschuss des Deutschen Bundestages teilgenommen (LPartG, LPartÜG, VersAusgIG, AGG, Gesetz zur Änderung des Zugewinnausgleichs- und Vormundschaftsrechts). Sie war Gutachterin für das nationale Forschungsprogramm „Kindheit, Jugend und Generationenbeziehungen im gesellschaftlichen Wandel“ des Schweizerischen Nationalfonds, für den österreichischen Fonds zur Förderung der wissenschaftlichen Forschung sowie die Deutsche Forschungsgemeinschaft. Von 2006 bis 2008 war Nina Dethloff Adhoc-Richterin am Europäischen Gerichtshof für Menschenrechte. In der Zeit von $\mathbf{2 0 0 6}$ bis 2011 war sie ferner Vorstandsmitglied der Zivilrechtslehrervereinigung und von 2008 bis 2010 Mitglied der Expertengruppe bei der Europäischen Kommission zum Güterrecht „Property consequences of marriage (PRM - III) in the European Union“. 2008 war Nina Dethloff Gutachterin für den 67. Deutschen Juristentag zum Thema „Sind unsere familienrechtlichen Ausgleichssysteme noch zeitgemäß? - Unterhalt, Zugewinn- und Versorgungsausgleich. 2012, im Rahmen des 69. Deutschen Juristentags, wurde sie von den Mitgliedern in seine Ständige Deputation gewählt. Außerdem ist sie im Vorstand der International Society of Family Law, Mitglied des American Law Institute und der Academia Europaea.

Nina Dethloff ist seit 2001 Mitglied des djb. 2005 hat sie anlässlich des 36. djb-Kongresses in Trier über „Diskriminierung im Familienrecht" vorgetragen.

Sie ist verheiratet und hat zwei Kinder. 
in denen wir engagiert mit den Professoren und Professorinnen diskutiert haben, konnten wir Verhandlungen vor dem Supreme Court miterleben oder einen Eindruck vom Gesetzgebungsprozess „on the hill“ bekommen. Meine in der Masterthesis zu einem aktuellen kartellrechtlichen Thema erworbene Expertise ermöglichte es mir, in der Folge eine Zeit lang unbekümmert für viele Monate mit VW-Bus und Rucksack unterwegs gewesen. Es beunruhigte mich nicht besonders, wenn es Aufstände gab, geschossen wurde oder hin und wieder auch ein Panzer vorbeirollte. Die Sicht der Eltern war bestimmt weniger entspannt, aber sie haben mich nie eingeschränkt.

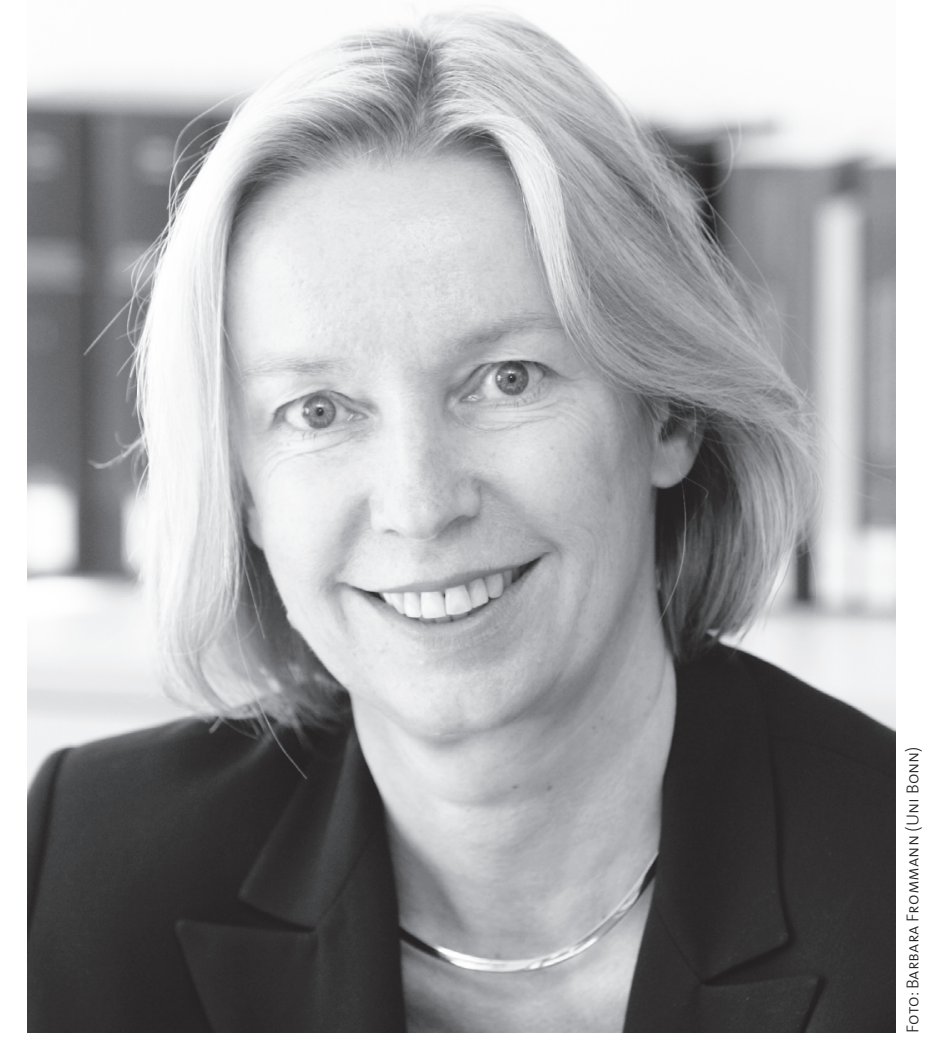

als Beraterin bei der Federal Trade Commission zu verbringen. Dass ich zugleich die Möglichkeit hatte, eine Zulassung als Rechtsanwältin in New York zu erlangen, war für mich ein besonderer Gewinn. Noch heute biete ich regelmäßig englischsprachige Veranstaltungen zum US-amerikanischen Recht an, in denen wir in einem „mock trial“ eine fiktive Gerichtsverhandlung durchspielen.

Mit 1. Staatsexamen und noch vor dem Referendariat als Rechtsanwältin in New York zu arbeiten, das wäre auch heute noch ein leicht exotischer Karriereverlauf. In die weite Welt hat es Sie später auch immer wieder gezogen, so haben Sie als Referendarin in einer Kanzlei in Buenos Aires gearbeitet. Sprechen Sie Spanisch?

Damals noch nicht gut genug, um Verhandlungen zu führen oder Rechtsgespräche, aber für normale Konversation reichte es. Mit der Wahl dieser Kanzlei hatte ich Glück, mein Ausbilder ließ mir sehr viel Freiraum und ich habe Einblick in viele Bereiche bekommen. Südamerika war ein spannender Kontinent im politischen Umbruch und nicht ganz ungefährlich, aber ich war schon als Studentin viel gereist und jugendlich
Sie haben schon vor dem Referendariat promoviert, zu einem familienrechtlichen Thema. Hatten Sie sich damit bereits für eine wissenschaftliche Laufbahn entschieden? Haben Ihre Lehrer Sie darin unterstützt?

Thematisch in Richtung meiner Doktorarbeit hatte ich in Freiburg schon ein Seminar gemacht, aber der Wunsch zu promovieren hat sich erst später entwickelt. Von besonderem Einfluss waren sicher meine Erfahrungen in Washington; dort habe ich auch die größte Offenheit und Unterstützung erfahren. Sie müssen sich vorstellen, in meiner gesamten Studienzeit in Deutschland habe ich nicht eine einzige Vorlesung von einer Frau erlebt, es gab schlicht keine Professorinnen in Jura. Auch heute ist mein Eindruck, dass es Frauen immer noch schwerer haben als Männer, in den Hochschulen Karriere zu machen. Die Mehrzahl der Professoren ist männlich, und sie suchen sich ihre Mitarbeiter und Nachfolger auch eher unter den Männern. Gemessen an dem hohen Anteil von Frauen an den Studierenden und erfolgreichen Absolventinnen irritiert schon die geringe Zahl weiblicher Promotionen. Hier muss unbedingt angesetzt und die Frauen motiviert und unterstützt werden. Dafür braucht es auch Rollenvorbilder, also vor allem Professorinnen. 
Ihre Vorstellungen von einer wissenschaftlichen Laufbahn haben Sie umsetzen können. Knapp zehn Jahre nach dem 2. Staatsexamen waren Sie habilitiert, hatten sofort einen Ruf an eine der renommiertesten deutschen Universitäten, nach Bonn, dem sie gefolgt sind, einen weiteren nach Hamburg, dem Sie nicht gefolgt sind, haben eine Familie gegründet und zwei Kinder bekommen. Wer oder was hat Sie auf diesem Weg unterstützend begleitet?

Da kommt einiges zusammen, und ein bisschen Glück war sicher auch dabei, aber ich hatte mich für eine wissenschaftliche Laufbahn entschieden und keinen Plan B in der Reserve; ich wusste, was ich wollte. Nach dem 1. Staatsexamen hatte ich meinen Mann kennengelernt, der ebenfalls eine wissenschaftliche Laufbahn anstrebte und mich in meiner Entscheidung stets bestärkt hat. Er war damals schon mit der Promotion befasst und daher auch früher als ich habilitiert. Nachdem im Abstand von zwei Jahren unsere beiden Kinder auf die Welt gekommen waren, sind wir zunächst dorthin gezogen, wo er seine erste Professur hatte. Aber mein Mann hatte mir zugesagt, dass die Familie später mir dahin folgen wird, von wo mich der erste Ruf erreicht. Im Vertrauen darauf habe ich neben der wissenschaftlichen Arbeit viel Zeit mit meinen Kindern verbringen können, worüber ich sehr froh bin, und mein Mann hat sein Versprechen auch gehalten. Als der Ruf kam, habe ich mich schweren Herzens gegen meine Heimatstadt Hamburg und leichten Herzens für die Rheinische FriedrichWilhelms-Universität in Bonn entschieden. Das war auch mit Blick auf unseren gemeinsamen Wohnsitz als Familie eine gute Wahl.

Bonn als langjähriger Sitz unseres Verbandes im mitgliederstarken Landesverband Nordrhein-Westfalen verfügt über eine sehr aktive Regionalgruppe. Was verbindet Sie mit dem djb?

Ich bin schon lange Mitglied im djb, und das ist mir auch sehr wichtig. Leider kann ich wegen anderer Verpflichtungen nicht regelmäßig zu den Veranstaltungen kommen, aber das schmälert nicht die Freude an der Gewissheit, über den Verband mit Frauen in Verbindung zu stehen, die die gleichen Interessen verfolgen und ihren Sachverstand und ihr rechtspolitisches Engagement bündeln. Alleine können wir nichts bewirken. Eine Aktion wie „Aktionärinnen fordern Gleichberechtigung“ mit dem Besuch der Hauptversammlungen börsennotierter Unternehmen finde ich ganz großartig. Solche Projekte sind wichtig und erfordern gemeinsame Initiativen, so etwas kann die Einzelne nicht schultern. Ich war selbst Aufsichtsrätin in einem börsennotierten Unternehmen, Vorsitzende des Verwaltungsrates des Studentenwerkes und bin Mitglied des Hochschulrates der Universität Osnabrück. Wir brauchen deutlich mehr Frauen in den Leitungsebenen von Unternehmen und Institutionen.

Neben Ihrer umfassenden Lehrtätigkeit und Mitgliedschaft in zahlreichen juristischen Gremien sind Sie auch Direktorin des Instituts für Deutsches, Europäisches und Internationa- les Familienrecht, sind als Sachverständige gefragt, Autorin die Liste Ihrer Aktivitäten ist lang. Jetzt kommt die Tätigkeit für den Deutschen Juristentag dazu: im September sind Sie in die Ständige Deputation des djt gewählt worden. 2008 waren Sie bereits als Gutachterin dabei. Was versprechen Sie sich von dieser neuen, zusätzlichen Aufgabe?

Der djt ist die Plattform, Rechtswissenschaft und Rechtspolitik zusammenzubringen und Themen zu setzen. Es freut mich sehr, daran in Zukunft mitwirken zu können, und wenn sich dadurch die Repräsentanz von Frauen in diesem wichtigen Gremium erhöht, dann freut es mich umso mehr, denn Recht und Gesetz beeinflussen die Lebenswirklichkeit von Männern und Frauen. Darum ist es nur folgerichtig, wenn sich beide Hälften der Bevölkerung in maßgeblichen Gremien abbilden.

Jenseits der nationalen Rechtsetzung gewinnt Europa immer mehr Einfluss auf unsere Gesetzlichkeit, auch im Familienrecht. So haben insbesondere die Urteile des EGMR zum Sorgerecht nichtehelicher Väter in jüngster Zeit für Unruhe gesorgt. Sie waren selbst Ad-hoc-Richterin am EGMR. Wie sehen Sie diese Entwicklung?

Ich begrüße diese Entscheidung des EGMR ausdrücklich als längst überfällig. Mit der bisherigen Regelung nimmt Deutschland eine Sonderstellung in Europa ein, die den heutigen Gegebenheiten nicht Rechnung trägt. Sie lässt das Kindeswohl außer Betracht.

Mir ist es ein zentrales Anliegen, dass Recht und Gesetz den Wandel gesellschaftlicher Verhältnisse in den Blick nehmen. Kinder wachsen heute in sehr unterschiedlichen Familienformen auf. Unabhängig davon, für welche Form des $\mathrm{Zu}$ sammenlebens sich ihre Eltern entschieden haben, bedürfen sie alle des Schutzes unserer Rechtsordnung.

Und auch die Paarbeziehungen sind heutzutage sehr vielfältig. Die Autonomie der Partner in der Gestaltung der Beziehung muss gewährleistet sein, das Recht muss aber zugleich Schutz bieten und entsprechende Rahmenbedingungen formulieren. Das ist hier seine Aufgabe: Freiheit und Schutz des Individuums zu gewährleisten.

Eine Masterarbeit zum Kartellrecht, eine Habilschrift zum Wettbewerbsrecht, promoviert haben Sie im Familienrecht, heute bildet das Familienrecht einen deutlichen Schwerpunkt Ihrer Arbeit - was meinen Sie: Bleibt das Familienrecht auch in Zukunft spannend?

Daran habe ich gar keinen Zweifel, im Gegenteil: mit der zunehmenden Globalisierung der Gesellschaft und den vielen grenzüberschreitenden Partnerschaften wird gerade das Familienrecht mit seinen vielfältigen rechtskulturellen Prägungen großen Herausforderungen gegenüberstehen. Im kommenden Jahr als Fellow des Käte-Hamburger-Kollegs werde ich vertieft Gelegenheit zur Beschäftigung mit diesen Fragen haben. Im Zentrum meiner Arbeit dort wird die Entwicklung eines optionalen Modells einer Europäischen Ehe stehen. Bei Wahl eines solchen einheitlichen Rechtsrahmens könnten Paare, deren Beziehung Berührungspunkte zu verschiedenen Rechts- 
ordnungen aufweist, von vornherein darauf vertrauen, dass ihre Ehe überall dieselben Wirkungen hätte. Für den Fall der Fälle wäre sie aber auch unter von vornherein vorhersehbaren Voraussetzungen und Folgen wieder auflösbar. Eine solche Europäische Ehe sollte so ausgestaltet sein, dass sie Paaren unterschiedlichster rechtskultureller Prägung einen ihren Bedürfnissen entsprechenden Rechtsrahmen bietet.

\section{Was ist Ihnen mit Blick auf die Studierenden besonders wichtig?}

Wir haben viele junge, begeisterte Menschen, die voller Enthusiasmus zu uns kommen. Es ist wichtig, diesen Nachwuchs zu fördern, und dabei auch die jungen Frauen in den Blick zu nehmen, sie zur Promotion zu ermutigen und ihren wissenschaftlichen Werdegang engagiert zu begleiten. Über die Gleichstellungsbeauftragte der Universität habe ich eine Anschubfinanzierung für Promotionen von Frauen erwirken können. Dieses Programm zeigt Erfolg. Die Zahl der Doktorandinnen ist in den letzten Jahren schon gestiegen. Und im Sommer 2011 hat sich die erste meiner Schülerinnen habilitiert. Darauf bin ich sehr stolz. Ich hoffe, dass noch einige folgen.

Unser weiteres Ziel muss es aber sein, die Frauen nicht nur vermehrt auf den Lehrstühlen zu sehen, sondern auch in der Leitungsebene der Universitäten, als Dekanin und Rektorin.
Als Vorsitzende meines Fachbereiches und Prodekanin bin ich gerade die einzige Frau in vergleichbarer Position im Haus. Das muss sich ändern.

\section{Hat sich für Sie alles so entwickelt, wie Sie es sich gewünscht haben - oder fehlt etwas?}

Meine berufliche Aufgabe macht mir sehr viel Freude. Mich den Themen intensiv widmen zu können, in die Tiefe zu gehen und in wissenschaftlicher Freiheit eigene Schwerpunkte setzen zu können, das ist es, was die Hochschullaufbahn auch heute für mich auszeichnet. Ich habe diese Wahl nie bereut. Es fehlt eigentlich nur ein bisschen mehr Zeit jenseits des fachlichen Engagements; früher habe ich viel gemalt, derzeit bleibt leider wenig Raum für einen Ausgleich.

\section{Liebe Frau Professorin Dethloff, ich danke Ihnen für dieses offene Gespräch.}

Sehr gerne! Ich freue mich auf die nächsten Begegnungen mit den Kolleginnen vom djb. Mit vielen von Ihnen werde ich vielleicht im nächsten Sommer zusammentreffen können: Vom 29. bis zum 31. August 2013 wird in Bonn die von der „Commission on European Family Law“ und der Universität Bonn veranstaltete Fünfte Konferenz zum Europäischen Familienrecht stattfinden. Die Vorbereitungen dazu laufen bereits auf Hochtouren.

\section{Impressum}

Schriftleitung

Anke Gimbal, Rechtsassessorin (V.i.S.d.P.)

Juliane Lindner

\author{
Redaktionsanschrift \\ Deutscher Juristinnenbund e. V. \\ Anklamer Str. 38 \\ 10115 Berlin \\ Telefon: 030 443270-0 \\ Telefax: 030 443270-22 \\ E-Mail: geschaeftsstelle@djb.de \\ www.djb.de
}

\section{Erscheinungsweise:}

4 Ausgaben im Jahr

\section{Bezugspreise 2012}

Jahresabonnement 52,- $€$; Einzelheft 14,- $€$.

Alle Preise verstehen sich incl. MWSt, zzgl. Vertriebskosten.

\section{Bestellmöglichkeit}

Bestellungen beim örtlichen Buchhandel oder direkt bei der Nomos Verlagsgesellschaft Baden-Baden

\section{Kündigungsfrist}

jeweils drei Monate vor Kalenderjahresende
Bankverbindung generell

Zahlungen jeweils im Voraus an Nomos Verlagsgesellschaft, Postbank Karlsruhe: BLZ 660 100 75, Konto Nr. 73636-751 oder Sparkasse Baden-Baden Gaggenau: BLZ 662500 30, Konto Nr. 5-002266

\section{Druck und Verlag}

Nomos Verlagsgesellschaft $\mathrm{mbH} \& \mathrm{Co}$. KG Waldseestr. 3-5, D-76530 Baden-Baden Telefon (07221) 2104-O/Fax (07221) 2104-27 E-Mailnomos@nomos.de

\section{Anzeigen}

Sales friendly Verlagsdienstleistungen

Siegburger Str. 123, 53229 Bonn

Telefon (0228) 978980, Fax (0228) 9789820

E-Mail roos@sales-friendly.de

Urheber- und Verlagsrechte

Die Zeitschrift sowie alle in ihr enthaltenen einzelnen Beiträge und Abbildungen sind urheberrechtlich geschützt. Jede Verwertung, die nicht ausdrücklich vom Urheberrechtsgesetz zugelassen ist, bedarf der vorherigen Zustimmung des Verlags.

Mit der Annahme zur Veröffentlichung überträgt der Autor dem Verlag das ausschließliche Verlagsrecht für die Zeit bis zum Ablauf des Ur- heberrechts. Eingeschlossen sind insbesondere auch das Recht zur Herstellung elektronischer Versionen und zur Einspeicherung in Datenbanken sowie das Recht zu deren Vervielfältigung und Verbreitung online oder offline ohne zusätzliche Vergütung. Nach Ablauf eines Jahres kann der Autor anderen Verlagen eine einfache Abdruckgenehmigung erteilen; das Recht an der elektronischen Version verbleibt beim Verlag.

Namentlich gekennzeichnete Beiträge geben nicht in jedem Fall die Meinung der Herausgeber/Redaktion oder des Verlages wieder. Unverlangt eingesendete Manuskripte - für die keine Haftung übernommen wird - gelten als Veröffentlichungsvorschlag zu den Bedingungen des Verlages.

Die Redaktion behält sich eine längere Prüfungsfrist vor. Eine Haftung bei Beschädigung oder Verlust wird nicht übernommen. Bei unverlangt zugesandten Rezensionsstücken keine Garantie für Besprechung oder Rückgabe. Es werden nur unveröffentlichte Originalarbeiten angenommen. Die Verfasser erklären sich mit einer nicht sinnentstellenden redaktionellen Bearbeitung einverstanden.

ISSN 1866-377X 